\title{
Pruebas de Provocación Bronquial con Ejercicio y con Histamina en Niños Asmáticos
}

\author{
Dr. Sergio Aguilar D.'; Dra. Daisy Lewisson N. ${ }^{1}$ \\ Dr. Rodrigo Moreno B. ${ }^{2}$; Dra. Camen Lisboa B. ${ }^{\frac{2}{2}}$ \\ Exercise and Fistamine challenges in children \\ with asthma
}

\begin{abstract}
Thirty one asthmatic children, 5 to 14 years old were studied, in order to compare exercise provocation test with histamine challenge for assesing aifway's hyperreactivity in asthma. Both tests were perfomed randomly at 48 hours intervals. Excercise consisted in an out-door 8 minutes nonstop running. $A 10 \%$ dectease in $F E V 1$, after exercise was considered positive. Histamine was administered by inhalation akong two minutes during tidal breathing in twofold increasing concentrations. A $20 \%$ dectease in FEV1 (Pc20) at histamine's concentrations of $8 \mathrm{mg} / \mathrm{ml}$ or higher was considered a normal response. There were no differences between FEV1, preceeding each challenge. Ninety seven percent of the patients had positive histamine test compared with only $74 \%$ or positive exercise test. A significant correlation was found between histamine Pc20 and the percent fall in FEV 1 after excercise $t r=0.404 \mathrm{p}<0,05)$. Pc20 histamine correlated significantly with basal FEV $1: \tau=0.464$ ( $\mathrm{P}<0,01$ ) but no correlation was found between basal and percent fall in FEVl aiter the exercise challenge. These results suggest that exercise is less sensitive than histamine challenge for screening airway's hyperreactivity in asthmatic children. (Key words: Asthma. Airway's hyperreactivity. Histamine provocation. Fxercise provocation).
\end{abstract}

La respuesta broncoconstrictora exgerada de la via aérea frente a diferentes estímulos inespe. cíficos constituye una de las características que definen el asma bronquial ${ }^{1}$. Por este motivo se han desarrollado diversas pruebas de provocación bronquial, con fines diagnósticos y de investigación ${ }^{2-3-4}$. El ejercicio físico es el estímulo broncoconstrictor que se emplea más frecuentemente en el niño ${ }^{4-5}$. Sin embargo su aplicación clínica tiene limitaciones derjvadas de su baja sensibili$\mathrm{dad}^{5-6-7}$ y de que la respuesta broncoconstrictora depende fundamentalmente de las condiciones de temperatura y humedad del aire ambiente inspirado ${ }^{8-10}$. Las pruebas de provocación bronquial con drogas broncoconstrictoras como histamina y metacolina, han sido, en cambio, utiiizadas sólo ocasionalmente en el niño a pesar

1 Servicio de Pediatría Hospital Josefina Martínez.

2 Departamento de Enfermedades Respiratorias. Fa. cultad de Medicina. Pontificia Universidad Católjca de Chile. que en el adulto han demostrado una buena sensibilidad y una caracterización muy objetiva de la hiperreactividad bronquial ${ }^{6-711}$.

Este estudio se realizó con el propósjto de comparar el rendimiento de las pruebas de provocación con ejercicio y con histamina en un grupo de niños con asma bronquial.

\section{MATERIAL Y METODOS}

Se estudiaron 31 niños con diagnóstico de asma bronquial, de acuerdo al criterio de la Sociedad Americana de Tórax ${ }^{1}$, que estaban en control $y$ tratamiento por alguno de los autores. 24 eran del sexo masculino y 7 del sexo femenino. La edad fluctuó entre 5 y 14 años con un pro. me dio de $9,20 \pm 2,72$. Sus características antro. pométricas se resumen en la Tabla 1.

El estudio se realizó en dos sesiones separadas por 48 horas: en una se midió la respuesta al ejercicio y en la otra la respuesta a la inhalación a la histamina. Estas pruebas fueron hechas en 
forma alternada, de modo que algunos niños iniciaron el estudio con ejercicio y otros con histamina.

El ejercicio consistió en una carrera al aire libre durante 8 minutos. La broncoconstricción se evaluó midiendo la caída del volumen espiratorio forzado del primer segundo $\left(V E F_{1}\right)$, antes del ejericicio y 5,10 y 15 minutos después de terminađo éste. El VEF, fue medido en un espirómetro Collins de 9 litros y los valores observados en condiciones basales fueron expresados en porcentajes del valor teórico ${ }^{12}$. En cada niño se realizaron por lo menos tres mediciones basales hasta asegurar una buena comprensión de la prueba. La magnitud del ejericicio realizado se valoró a través de la frecuencia cardíaca alcanza. da al final de los 8 minutos.

La prueba de provocación con histamina se realizó utilizando una modificación del método, propuesta por Cockroft y col. ${ }^{2 \cdot 11}$. El diluyente de la histamina y la solución de fosfato ácido de histamina se administraron por aerosol durante 2 minutos de respiración tranquila a través de la boca, utilizando un nebulizador de Hudson que, con un flujo de 5 litros por minuto, suministra $0,3 \mathrm{ml}$ por minuto en particulas con una masa aerodinanica media de $2 \mu \mathrm{m}$. El nebulizador unido a una mascarilla se aplico a la cara del niño. La prueba se inició con el registro basal del VEF $\mathrm{VE}_{1}$, medido por lo menos tres veces una vez asegurada una buena comprensión de la prueba. Posteriormente se administró el diluyente de la his- tamina por aeorosol durante dos minutos y las determinaciones del $V E F_{1}$, se hicieton al tercer minuto. El valor más bajo obtenido después del diluyente se útilizó como $V F_{1}$ de referencia. Se excluyó del estudio aquellos niños en que el VEF $_{1}$ descendió más de $10 \%$ en relación al valor basal después de la inhalación del diluyente. Luego se administró el fosfato ácido de histamina comenzando con una concentración inicial de $0,06 \mathrm{mg} / \mathrm{ml} \mathrm{y}$ midiendo nuevamente $\mathrm{VEF}_{1}$ al tercer minuto de iniciada la nebulización. La dosis đe histamina se aumentó progresivamente duplicando la concentración. La prueba se suspendió en la concentración inmediatamente superior a la que produjo $20 \%$ de caida del VEF $\left(\mathrm{Pc}_{20}\right)$.

Con los datos de cada paciente se construyó una curva de dosis-respuesta en la que se puso $V F_{1}$ en el eje vertical, expresado como porcentaje del valor obtenido con el diluyente $y$ en el eje horizontal las concentraciones de histamina en escala logarítmica. En cada curva se cátculó [a $\mathrm{Pc}_{20}$ o dosis umbra], que es la concentración de histamina que produce $20 \%$ de la caída del $V F_{1}$. En la figura l se ilustra una curva de respuesta representativa en la que se indica ia $\mathrm{Pc}_{20}$.

En 22 de los nifios ambas pruebas se realizaron con $V E F_{1}$ basal superior al $70 \%$.

En 9 riños el VEF 1 fue superior a $70 \%$ : en 3 , en la prueba de histamina, en 2 en la de ejercicio y en 4 en ambas pruebas (Tabla 1 ).

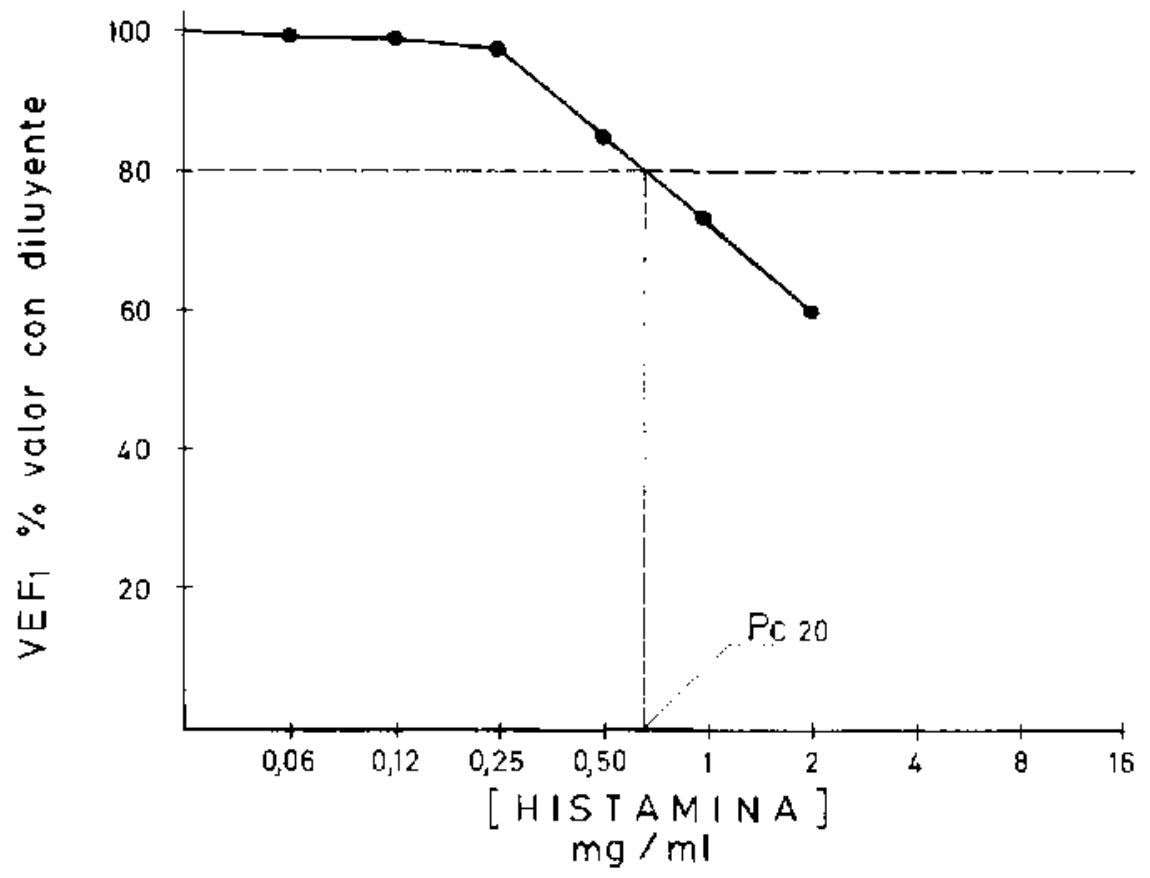

Figura 1.

Curva dosis respucsta a histamina obtenjida en un paciente.

La línea vertical discontinua muestra la concentración que corresponde a la $\mathrm{Pc}_{2} 0$. 
Tabla !

Caracter ísticas de los niños estudiados

\begin{tabular}{|c|c|c|c|c|c|c|c|c|}
\hline Caso & Sexo & $\begin{array}{l}\text { Edad } \\
\text { Años }\end{array}$ & $\begin{array}{l}\text { Talla } \\
\text { cm }\end{array}$ & $\begin{array}{l}\text { Atopia } \\
\text { Cutánea }\end{array}$ & Tratamiento & $\begin{array}{c}\text { VEFl H } \\
\text { \& teórico }\end{array}$ & $\begin{array}{l}\mathrm{VEF}_{1} \mathrm{E} \\
\% \text { teórico }\end{array}$ & $\begin{array}{c}\text { Frec. } \\
\text { Cardíaca } \\
\text { L } x\end{array}$ \\
\hline 1 & $\mathrm{~F}$ & 5 & 119 & + & B.D. diario & 69 & 73 & 180 \\
\hline 2 & $\mathbf{M}$ & 5 & 112 & - & B.D. diario & 98 & 96 & 178 \\
\hline 3 & $\mathbf{M}$ & 5 & 123 & + & B.D. SOS & 87 & 82 & 198 \\
\hline 4 & $\mathbf{M}$ & 6 & 125 & + & B.D. SOS & 79 & 83 & 186 \\
\hline 5 & $\mathbf{M}$ & 6 & 120 & + & B.D. diario & 72 & 80 & 176 \\
\hline 6 & $\mathbf{M}$ & 7 & 128 & + & B.D. SOS & 75 & 82 & 196 \\
\hline 7 & $\mathbf{M}$ & 7 & 125 & + & B.D. diario & 99 & 103 & 168 \\
\hline 8 & $\mathbf{M}$ & 7 & 122 & + & sos & 98 & 84 & 202 \\
\hline 9 & $F$ & 7 & 125 & + & B.D. diarjo & 71 & 91 & 198 \\
\hline 10 & $\mathbf{M}$ & 7 & 134 & + & B.D. diario & 74 & 71 & 190 \\
\hline 11 & $\mathrm{~F}$ & 8 & 129 & $?$ & $\operatorname{Sin}$ & 85 & 74 & 178 \\
\hline 12 & $\mathbf{M}$ & 8 & 137 & + & SOS & 79 & 55 & 198 \\
\hline 13 & $\mathbf{M}$ & 8 & 123 & - & SOS & 94 & 83 & 189 \\
\hline 14 & $\mathbf{M}$ & 9 & 131 & + & SOS & 81 & 75 & 196 \\
\hline 15 & $\mathbf{M}$ & 9 & 131 & + & B.D. diario & 72 & 72 & 177 \\
\hline 16 & $\mathbf{M}$ & 9 & 125 & - & $\operatorname{Sin}$ & 97 & 86 & 187 \\
\hline 17 & M & 10 & 150 & + & B.D. diario & 56 & $6 B$ & 169 \\
\hline 18 & $\mathrm{~F}$ & 10 & 138 & + & B.D. diario & 81 & 78 & 188 \\
\hline 19 & $\mathrm{~F}$ & 10 & 141 & $?$ & $\operatorname{Sin}$ & 67 & 72 & 176 \\
\hline 20 & $\mathbf{M}$ & 11 & 136 & + & SOS & 66 & 71 & 186 \\
\hline 21 & $\mathrm{~F}$ & 11 & 142 & + & sos & 99 & 97 & 166 \\
\hline 22 & $\mathbf{M}$ & 11 & 140 & + & $\begin{array}{l}\text { B.D. diario } \\
\text { Corticoides }\end{array}$ & 66 & 53 & 198 \\
\hline 23 & $\mathbf{M}$ & 11 & 135 & - & $\mathrm{Sn}$ & 87 & 103 & 198 \\
\hline 24 & $\mathrm{~F}$ & 11 & 137 & - & B.D. diario & 85 & 78 & 198 \\
\hline 25 & $\mathbf{M}$ & 11 & 139 & - & $\sin$ & 63 & 63 & 196 \\
\hline 26 & M & 12 & 164 & + & sos & 78 & 69 & 189 \\
\hline 27 & $\mathbf{M}$ & 12 & 166 & + & B.D. diatio & 103 & 88 & 172 \\
\hline 28 & $\mathbf{M}$ & 13 & 149 & + & B.D. diario & 49 & 66 & 200 \\
\hline 29 & $\mathbf{M}$ & 14 & 158 & + & B.D. diario & 90 & 97 & 172 \\
\hline 30 & $\mathbf{M}$ & 14 & 159 & + & sos & 90 & 79 & 168 \\
\hline 31 & $\mathbf{M}$ & 14 & 159 & $?$ & Sin & 84 & 86 & 152 \\
\hline $\mathrm{X}$ & & 9,29 & 135,9 & & & 80,45 & 78.97 & 184,58 \\
\hline $\mathrm{DS} \pm$ & & 2,72 & 14,0 & & & 13,6 & 12,5 & 12,7 \\
\hline
\end{tabular}

VEF 1 H Vohmen espiratorio del primer segundo previo a la prueba de histamina.

$\mathrm{VEF}_{1 \mathrm{E}}=\mathrm{VEF}_{1}$ previo a la prueba de ejercicio. B.D. Diarios Beta adrenérgicos o aminofilina diaria. SOS Beta adrenérgicos o aminofilina ocasional.

Sin: Sin tratamiento.

En los días de estudio se suspendió la medicación antiasmática: 12 horas antes los derivados de teofilina y 8 horas, los betaadrenérgicos. No se suspendieron los corticoides.

Los padres y los niños fueron completamente informados y aceptaron participar en el estudio.

Cono límite normal de dosis umbral de hista. mina se considera $8 \mathrm{mg} / \mathrm{ml}$. Esta valor es el correspondiente a adultos normales estudiados con el mismo método en nuestro laboratorio ${ }^{11}$ y a los propuestos por Cockcroft y cols. ${ }^{2}$. La prueba de ejercicio fue calificada como positiva si la caída máxima del $V_{E F}$ después del esfuerzo superaba el $10 \% 4$.

Los resultados se analizaron mediante pruebas de student para muestras pareadas y regresión lineal ${ }^{13}$.

\section{RESULTADOS}

En la figura 2 se ilustran los valores individua- 
les de da $\mathrm{Pc}_{20}$ de histamina y del porcentaje de caída del $V E F$ post ejercicio observado en los pacientes. La linea horizontal limita el rango normal determinado en nuestro laboratorio utilizando el mismo método. Se puede observar que sỏlo un niño tuvo dosis umbral normal. El valor promedio fue de $1.37 \mathrm{mg} / \mathrm{ml} \pm 1,8$ D.S. El promedio de caída de $V F_{1}$ post ejercjcio fue de $21,37 \% \pm 17,32$. En 8 de los 31 niños el VEF no cayó significativamente con el ejercicio.
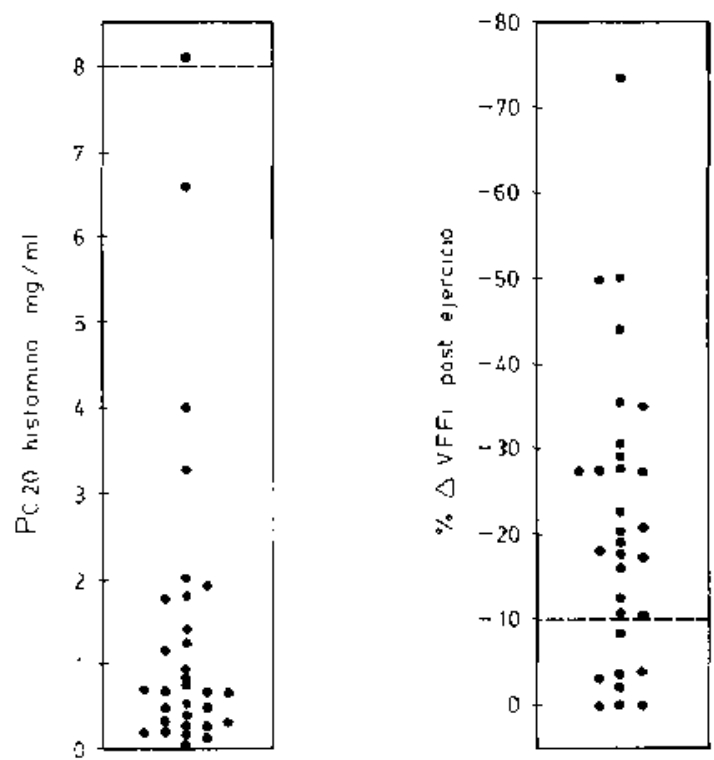

Figura 2.

Valotes individuales de $\mathrm{PC}_{2} \mathrm{O}$ histamina (izqujerda). Porcentaje de caida del VE.F 1 post ejercicio (derecha). Cada punto corresponde a un paciente. La linea horizontal señala límites de normalidad.

En la Figura 3 se muestra la relación entre la máxima caída del $V E F_{1}$ post-ejercicio, en el eje horizontal, y la dosis umbral de histamina, en el eje vertical. El coeficiente de correlación obtenido fue de $0,408(p<0.05)$. Se observa que el niño que tenia $\mathrm{Pc}_{20}$ nomal de histamina $(8 \mathrm{mg} / \mathrm{ml})$ tampoco se obstruyó con el ejercicio. En cambio 7 de los 8 niños que no se obstruyeron con el ejericicio tuvieron dosis umbral de histamina baja, entre 0,5 y $6,6 \mathrm{mg} / \mathrm{ml}$.

Se encontró una relación significativa $(r=0.464 p<0,01)$ entre el VEF 1 basal previo a la provocación con histamina y la $\mathrm{Pc}_{20}$ (Fig. 24). En cambio no hubo correlación entre el $V_{E F}$ antes del esfuerzo y su cajda con el ejercicio ( $\mathrm{r}=0.161$ p .VS) (Fig. 5).

No encontramos diferencias significativas entre los valores de $\mathrm{VEF}_{1}$ previos a histamina y a ejercicio.

No se observaron relaciones entre la magnitud de la hiperreactividad a la histamina y al ejercicio, con medicación utilizada para controlar los sintomas de asma, ni con la edad de los niños.

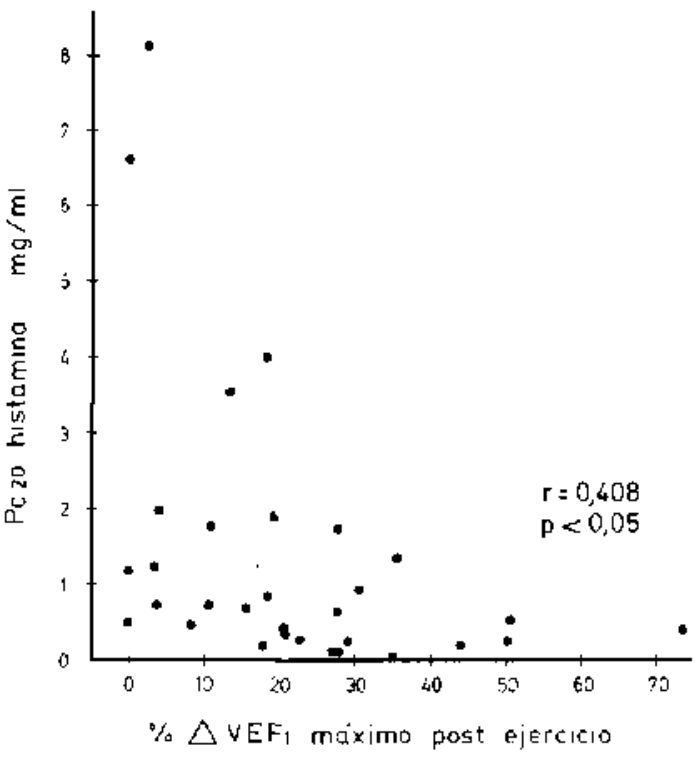

Figura 3

Relación wntre Pco histamisla (eje vertical) y VEF 1 previo a la prueba con histamina (eic horizontal).

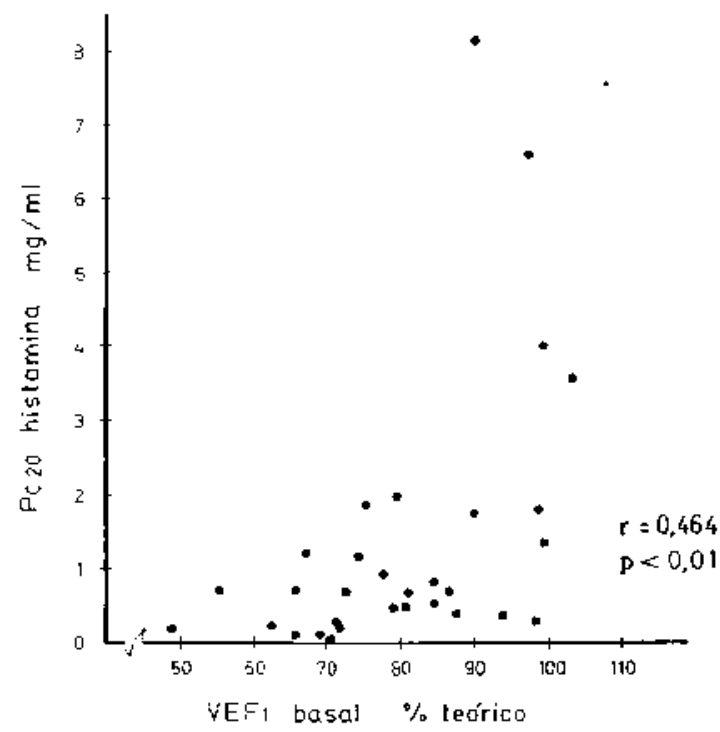

Figura 4.

Relación entre $\mathrm{P}_{\mathrm{c}_{20}}$ histamina (eje vertical) y $\mathrm{VH} \mathrm{F}_{1}$ post ejercicio (eje horizontal) (ver texto) 


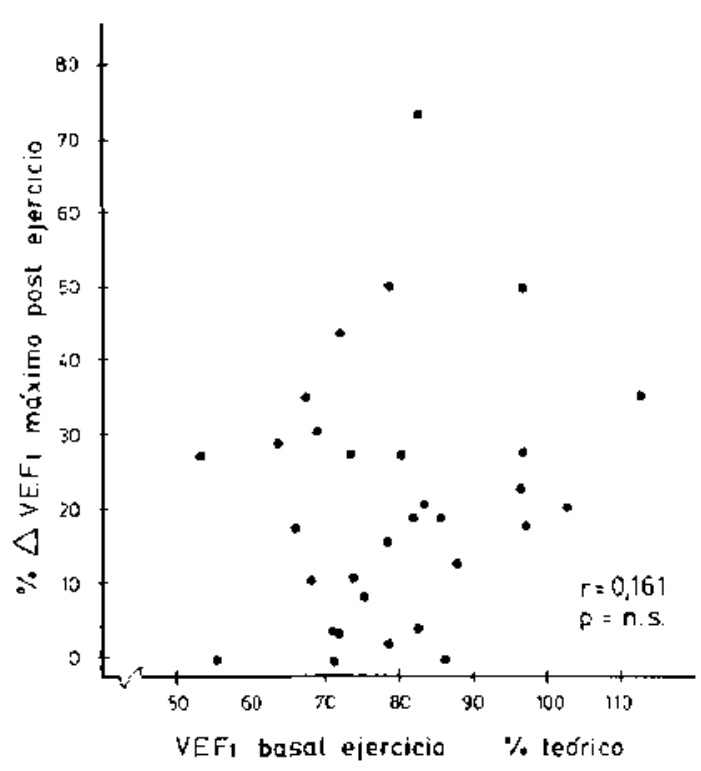

Figura 5.

Relación entre caida del VEF 1 post ejercicio (eje vertical) y $V F_{1}$ previo ejercicio (eje horizontal).

\section{DISCUSION}

Estos resultados demuestran que la prueba de provocación bronquial con histamina es más sensible que la de ejercicio en el diagnóstico de asma en el niño, ya que $97 \%$ de los enfermos tuvo disminución de $\mathrm{P}_{2} 0$ con histamina y sólo $74,2 \%$ prueba positiva con el ejercicio, aun cuando se exigió una cajda del VEF 1 del $10 \%$ : Si se hubiera considerado positiva una disminución del $20 \%$ del VEF prueba de histamina, sólo $48,4 \%$ de los pacientes estudiados tendrían prueba de esfuerzo positiva. Observaciones similares se han hecho en adultos: Anderton y cols. en $1979^{6}$ concluyeron que la prueba de histamina era superior al ejercicio para demostrar un aumento de la reactividad bronquial inespecífica. Chatman y cols. ${ }^{7}$ han demostrado, recientemente, que las pruebas de histamina y metacolina permiten diferenciar mejor a los normales de los asmáticos que la prueba de ejercicio. En niños,Mellis y cols ${ }^{5}$ utilizando la técnica de dosis acumulativa de histamina y el esfuerzo en cinta rodante, comunican $90 \%$ de resulta. dos positjvos con histamina y $74 \%$ con ejercicio.

Dado que en los estudios con histamina publi. cados en niños se usa un método diferente ${ }^{5-14}$, no existe información sobre la dosis umbral nor. mal. Por ello, presumimos un valor igual que el de los adultos, que es, en nuestra experiencia, de $8 \mathrm{mg} / \mathrm{ml} \mathrm{y}$ en la de Woolcock ${ }^{15}$ de $5 \mathrm{mg} / \mathrm{ml}$. Si consideramos como normal esta última cifra el porcentaje de positividad de la prueba de histamina en nuestros casos descenderá de 97 a $94 \%$.

A diferencia de lo observado por Mellis y cols. ${ }^{5}$, en el presente estudio se encontró una correlación significativa entre el $V_{E F}$ basal y la dosis umbral de histamina lo que significa que, como grupo, los niños que fueron estudiados con mayor obstrucción bronquial inicial presen. taron una hiperreactividad mayor. Este hallazgo confirma la conveniencia de realizar las pruebas de provocación con $V F_{1}$ basal superior al $70 \%$ del teórico con el propósito de no sobrevalorar la reactividad bronquial ${ }^{2-11}$

En 9 de los ninos del presente estudio esta exigencia no pudo ser cumplida y la prueba se realizó con $V E F_{1}$ basal inferior a $70 \%$ : en 4 en ambas pruebas y en 5 en la segunda de ellas. $\mathrm{Si}$ bien esto haría objetable estos resultados, cabe destacar que no se observó una tendencia sistemática a una menor $\mathrm{Pc}_{20}$ a una mayor caida del VEF, en aquéllos con menor VEF, basal.

La falta de correlación del $V F_{1}$ previo al ejercicio y la caida máxima del $V_{E F}$ post-ejercicio observada en nuestros pacientes también difiere de lo comunicado por Mellis y col. ${ }^{5}$ quienes encontraron que el flujo espiratorio forzado entre el 25 y $75 \%$ de la CV $\left(\right.$ FEF $\left._{25-75}\right)$ de los niños que se obstruyeron con ejercicio era significativamente menor que el de los que no se obstruyeron. Esta discordancia podía deberse, por una parte, a que estos autores utilizaron para valorar la obstrucción el $\mathrm{FEF}_{25-75}$ que es más sensible que el VEF 1 y, por otra parte, al diferen. te tipo de ejercicio realizado, pues los pacientes de Mellis y cols. lo realizaron durante 6 minutos, en una cinta rodante ubicada dentro de un edificio $^{5}$.

En los últimos años se ha logrado establecer que el estímulo que desencadena la broncoconstricción después del ejericicio es el enfriamiento y la desecación de la vía aérea por la hiperventilación, de modo que no se requiere de la actividad corporal en sí misma para provocar la broncoconstricción ${ }^{16-17}$. Esta dependencia de las condiciones físicas del aire inspirado hace necesario conocer la magnitud de la ventilación, la temperatura y la humedad del aire inspirado para comparar adecuadamente pruebas de esfuerzo, factores que no fueron medidos en el presente estudio como tampoco en el de Melliss, pudiendo explicar algunas de las diferencias observadas.

Como indice de la intensidad del ejercicio físico se utiliza la frecuencia cardiaca. Esta debe alcanzar cifras cercanas a los $180 / \mathrm{min}^{4-5-18}$. En nuestros pacientes se logró un promedio de $184,58 \pm 12,7$ latidos por minuto. De los 5 niños 
que alcanzaron frecuencia cardiaca inferior a 170 latidos por minuto sólo 1 no sufrió obstrucción y por otra parte, 2 de los 12 niños con frecuencias cardíacas iguales o superiores a 190 latidos por minuto tampoco tuvieron obstrucción bronquial postejercicio. Estos hallazgos hacen improbable que una insuficiente intensidad del ejercicio sea responsable de su menor sensibilidad como prueba de reactividad bronquial.

Una de las limitaciones de la prueba de es. fuerzo es la imposibilidad de controlar la magnitud de la obstrucción provocada que, con alguna frecuencia, puede alcanzar nivcles muy acentuados. En el presente estudio 3 niños tuvieron una caida del $V E F_{1}$ superior al $50 \%$ acompañada de importante disnea. Además, la suma del agotamiento físico y de la disnea puede llegar a ser muy inconfortable.

La prueba de histamina en incrementos sucesivos, tiene la ventaja de que produce bronco. constricción en forma progresiva y controlable, pudiendo, suspenderse la prueba cuando se rcgistra una caida moderada del $V_{E F} 1$ que, generaimente, no se acompaña de disnea muy importante. Además, el efecto de la histamina es transitorio $y$ las molestias secundarias aparecen, generalmente, con concentraciones altas. En nuestro estudio dos niños experimentaron cefalea; tres eritema facial transitorio $y$ dos broncoespasmo suficientemente acentuado coma para tener que emplear broncodilatadores.

Las pruebas de provocación con drogas dependen del grado de obstrucción bronquial inicial ${ }^{19}$ y de la gravedad del asma valorada a través de la medicación utilizada para controlar los sintomas ${ }^{2-1} 1-20$. Entre los factores inherentes al método mismo el más importante es la cantidad de drogas inhalada ${ }^{21}$ la que depende fundamentalmente del suministro que de el nebulizador. Por este hecho es imprescindible usar siempre nebulizadores de entrega similar.

La me nor sensibilidad del ejercicio en relación a la histamina en nuestros casos no puede atribuirse a diferencias en el grado de obstrucción previo a la realización de ambas pruebas pues el $V F_{1}$ inicial fue semejante.

Si bien la provocación con histamina dio resultados positivos con más frecuencia que el ejercicio, en uno de los pacientes ambas pruebas fueron negativas. En estos casos es posible que la provocación mediante hiperventilación con aire frio y seco pudiera ser más sensible 22 . Anderton y col. ${ }^{6}$ han comunicado que la prueba de ejercicio es negativa si la dosis umbral de histamina es superior a $3 \mathrm{mg} / \mathrm{ml}$. En el presente estudio. 2 de los niños oon dosis umbral superior a $3 \mathrm{mg} / \mathrm{ml}$ tuvieron prueba positiva de ejercicio por.lo que no nos es posible adoptar esta rela- ción entre el valor de la $\mathbf{P c}_{20}$ y la positividad de la prueba con ejercicio

En resumen, la curva dosis respuesta obtenida por inhalación de histamina es más sensible que el ejercicio para el diagnóstico de asma en el niño $y$ tiene las ventajas de ser simple, bien tole. rada y no presentar riesgos.

\section{RESUMEN}

Con el propósito de comparar el rendimiento de las pruebas de provocación bronquial con ejercicío fúsico y con histamina en el diagnóstico de asma bronquial, se estudiarón 31 niños asmáticos entre 5 y 14 años. El ejercicio consistió en carrera al aire libre durante ocho minutos. La histamina se adtministró por aerosol durante respiración tranquila. La obstrucción bronquial post-ejercicio se midió con la máxima caida del $V \mathrm{EF}_{1}$ a los 5,10 y 15 minutos después del esfuerzo. Se consideró positiva una caída máxima superior al 10\%. En la prueba de histamina se calculó la $\mathrm{Pc}_{20}$ o concentración de histamina que produce un $20 \%$ de caida del $V F_{1}$. En $97 \%$ de los niños disminuyó la $\mathrm{Pc}_{20}$ con hista. mina ( $\bar{x} 1,37 \mathrm{mg} / \mathrm{ml} \pm 1,8$ DS) comparado con sólo un $74 \%$ de reducciones significativas del $V F_{1}$ post ejercicio ( $\bar{x} 21,37 \pm 17,32 D S$ ).

Se encontró una correlación significativa $r=0,408 \mathbf{p}<0,05$ entre $P_{c_{20}}$ histamina y máxima caída $\mathrm{VEF}_{1}$ post-ejercicio. La $\mathrm{P}_{\mathrm{C}_{20}}$ histamina se relacionó significativamente con el $V F_{1}$ previo a la prueba $(\mathrm{r}=0,464 \mathrm{p}<0,01)$ ел cambio la caída del $V E F_{1}$ post ejercicio no estuvo relacionada con el VEF 1 previo $(r=0,161)$.

Los resultados demuestran el mayor rendimiento de la prueba de histamina comparada con el ejercicio en el diagnóstico de asma bronquial en el niño, $y$ sugieren que este último no es un buen indice para demostrar heperreactividad bronquial en el niño.

\section{REFERENCIAS}

${ }^{1}$ American Thoracic Saciety. Commitee of diognostic standards for non-tuberculous disease. Definition and classification of chronic bronchitis, asthma and pulmonary emphysema. Am. Rev. Respir. Dis. 85: 762, 1972 .

${ }^{2}$ Cockeroft D.W., Killian D.N., Mellon J.J.A., Hargreave $F . E$. Bronchiol reactivity to inhaled histami. ne: a clinical survey. Clin Allergy 7: 235, 1977

${ }^{3}$ Chai H., Farr R.S., Froehlich L.A., Mathison D.A. McLeon J.A., Rosenthal R.R., Sheffer A.L., Spector S.L., Townley R.G. Standardization of bronchial inhalation challenge procedures. J Allergy Clin Immunol 56; 323, 1975.

4 silvemen M. Anderson S.D. Standatdization of exercise tests in asthmatic children. Arch Dis Child 74: 882, 1972. 
$\$$ Mellis C.M., Kattan M., Keens T.G., Levison H. Comparative study of histamine and exercise challenges in asthmatic children. Am Rev Respir Dis 117:991, 1978.

'Anderton R.C., Cuff M.T., Frtth P.A., Cockcroft D.W.. Morse J.L.C., Jones N.L., Hargreave F.E. Bronchial responsiveness to inhaled histamine and exercise. J Allergy Clin Immunol 63: 315, 1979.

7 Chathom M., Bleecker E,R, Smith P.L., Rosenthal R.R., Moson P., Norman P.S. A comparison of histamine, methacholine, and exercise airway reactivity in normal and asthmatjc subjects. Am Rev Respir Dis 126: 235, 1982.

8 Bar-Or O., Neuman I., Dotan R. Effects of dry and humid climates on exercise-induced asthma in children and preadolescents. I Allergy Clin Immunol 60: 163,1977 .

9 Strauss R,H, McFadden E.R., Ingram R.H., Jaeger $J . J$. Enhancement of exercise-induced asthma by cold air. New Engl J Med 297: 743, 1977.

10 Strauss R.H., McFadden E.R., Ingrom R.H., Deal $E . C$. Joeger J.J. Influence of heat and humidity on the airway obstruction induced by exercise in asthma, J Clin Invest 61: 433, 1978.

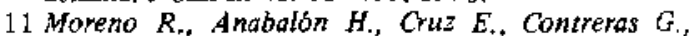
Lisbaa $C$. La curva de Iespuesta a la histamina en el diagnóstico y tJatamiento del asma bronquial. Rev Med Chile 110: 426, 1982 .

12 Cerecedo J., Atisha J., Calleja O., Cuz E, Lisboa C. Valores normales de flujos espiratorios máximos en niños. Rev Med Chile 104: $519,1976$.

13 Snedecor G.W., Cochrone W,G. Statistical methods. 6th ed. Jowa. The iowa State University Press, 1967.

14 Bhagot R., Grunstein M.M. Comparison of responsiveness to methacholine, histamine, and exercise in stubgroups of asthmatic childen. A.m Rey Respir Dis 129: $221,1984$.
15 woolcock A.J., Dowse G.K., Temple K., Stanley H.,

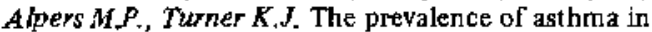
the South-Fore people of Papua New Guinea. A method for field studies of bronchial reactivity. Eur J Respir Dis 64: 571, 1983.

16 Deal E.C., McFadden E.R., Ingram R.H., Strauss R.H., Jaeger $J$.J. Role of respiratory heat exchange in production of exercise-induced asthma. J Appl Physiol: Respirat Environ Exercise Physiol 46: 40\%, 1979.

17 Zeballos R.J., Shturman-Ellstein R., McNally J.F., Hirsch J.E., Souhrada J.F. The role of hyperventilation in exercise-induced bronchoconstriction. Amer Rev Respir Dis 118: 877, 1978.

${ }^{18}$ Nejjens H.J., Duiverman E.J., Kerrebijn K.R. Bronchial Responsiveness in Children. Pediatric Clin North Amer 30: 829, 1983.

19 Chung K.F., Morgan B., Keyes S.J., Snashall P.D. Histaminc dose-response relationshlps in normal and asthmatic subjects. The importance of starting airway caliber. Am Rev Respir Dis 126: 849, 1982.

20 Juniper E.F., Frith P.A., Hargreave F.E. Airway Responsiveness to histamine and methacholine. Relationship to mininum treatment to control symptoms of asthma. Thorax 36:575, 1982.

${ }^{21}$ Ryan G., Dolovich M.B., Obmisnki G., Cockcroft D.W. Juntper E. Hargreave F.E., Newhouse M.T. Standardization of inhalation provocation tests: inlluence of nebulizer output, particle size, and method of inhalation. J Allergy Clin Immunol 67: 156,1981 .

22 Deal E.C., McFadden E.R., Ingram R.II, Breslin F.J., Jaeger J.J. Airway responsiveness to cold air and hyperpnea in normal subjects and in those with hay fever and asthma. Am Rev Respir Dis 121: 621,1980 . 